\title{
Constraining theories of low-scale quantum gravity by non-observation of the bulk vector boson signal from the Sun
}

\author{
R. Horvat, D. Kekez, Z. Krečak and A. Ljubičić \\ Rudjer Bošković Institute, P.O.B. 180, 10002 Zagreb, Croatia
}

\begin{abstract}
In this experiment we aim to detect Kaluza-Klein (KK) excitations of the bulk gauge field, emitted in a bremsstrahlung process on solar plasma constituents, by looking at a process analogous to the photoelectric effect inside the HPGe detector. Using a generic feature of the underlying effective theory that the unknown 4-dimensional gauge coupling is independent of the number of extra large dimensions $\delta$, we show that the expected number of events in the detector is insensitive to the true scale of quantum gravity for $\delta=2$. With the entire data collection time of 202 days in the energy interval $1.7-3.8 \mathrm{keV}$, the number of events detected was as low as $1.1 \cdot 10^{6}$, compared to $2.7 \cdot 10^{6}$ from the expected high multiplicity of the solar KK excitations for $\delta=2$. Hence, our bound from the presumed existence of new forces associated with additional gauge bosons actually conforms with very stringent bounds set from various astrophysical considerations. Baring any modifications of the infrared part of the KK spectrum, this bound would therefore rule out the possibility of observing any signal at the LHC for $\delta=2$. Although a dependence on the fundamental scale referring to $(4+\delta)$-dimensional gravity turns on again for $\delta=3$, the experimental sensitivity of the present setup proves insufficient to draw any constraint for $\delta>2$.

PACS numbers: 04.50.-h; 12.60.Cn; 32.80.Fb
\end{abstract}

In a remarkable proposal of Arkani-Hamed, Dimopoulos and Dvali (ADD) 1], the scenario of large extra dimensions has been advocated as an alternative viewpoint capable to shed some light on the gauge hierarchy problem . The radical revision from the standard description of physics beyond SM lies in the fact that low-scale (right above the weak scale) Planck mass $M_{*}$ and Newton's constant $M_{P l}^{-2}$ do peacefully coexist, if gravity is allowed to propagate in $\delta$ extra large compact dimensions. By applying Gauss' law, the simple case of the original Kaluza-Klein (KK) theory [2] can be generalized to $\delta$ extra dimensions as

$$
M_{P l}^{2}=M_{*}^{2+\delta} V_{\delta},
$$

where, considering a $\delta$-dimensional torus of radius $R, V_{\delta}=(2 \pi R)^{\delta}$.

Although the ADD proposal is considered only as a reformulation of the hierarchy problem and not a full solution ${ }^{1}$, it is still of non-ceasing interest nowadays due to its remarkable phenomenological implications [4]. Besides bulk gravitons, the same scenario, in which heavy mass scales in four dimensions are replaced by lighter mass scales in higher dimensions, can be applied to other non-SM fields as well. Examples cover successful application to neutrino [5] as well as axion phenomenology [6]. Since the SM has been tested down to distances $10^{-16} \mathrm{~cm}$, much shorter than the compactification radius $\mathrm{R}$, such class of higher-dimensional theories is nowadays conventionally considered in the context of the brane paradigm, where all the SM degrees of freedom are localized on a 3-brane. The realization of the ADD proposal is possible in type I string theory [7], for example, with the SM degrees of freedom localized on a D-brane.

On the observational side, there are two main classes of laboratory tests for the ADD proposal. The first searches for possible deviations from Newton's inverse-squared law in table top experiments [8]. Typically, we have $M_{*}>2$ $\mathrm{TeV}$ for $\delta=2$, although model-dependent effects can substantially affect these predictions. The second class deals with the collider bounds, giving $M_{*}>1.45(0.65) \mathrm{TeV}$ for $\delta=2(6)$ for the combined LEP-Tevatron data [9], with an extra possibility to study the so-called transplanckian regime at the LHC [10]. Much more stringent bounds on gravity in flat extra dimensions comes from variety of astrophysical considerations. The most restrictive limits on large extra dimensions comes from low measured luminosity of some pulsars, giving $M_{*}>750$ (35) TeV for $\delta=2$ (3) 11]. However, contrary to the collider bounds, these bounds are quite sensitive to the infrared part of the KK graviton spectrum.

Yet another possibility is to have gauge fields in the bulk, gauging $B-L$ (or either $B$ or $L$ separately), for example [4]. The limited impact of additional gauge bosons at low energy, due to decoupling of heavy-particle species, may not be the case any more if the new bosons are allowed to propagate freely in the bulk. The one-loop correction of a bulk gauge boson to the muon magnetic moment has already been calculated [12]. In the present paper, we aim to observe

\footnotetext{
${ }^{1}$ Instead of explaining why the Higgs VEV is small, one now needs to explain why $V_{\delta}$ is huge compared with $M_{*}^{-\delta}$. Moreover, in this scenario the hierarchy problem becomes closely related to the cosmological constant problem [3].
} 
gauge-boson KK excitations coming from the Sun and emitted in a bremsstrahlung process, $e+X \rightarrow e^{\prime}+X+b$, where $\mathrm{X}$ is a proton or $\alpha$ particle and " $b$ " stands for the KK gauge boson. Our experimental setup has been designed such as to capture KK modes from the Sun in the HPGe detector, in a process analogous to the ordinary photoelectric effect, $b+A \rightarrow e+A^{\prime}$, where now $A\left(A^{\prime}\right)$ refers to the germanium atom. Due to the expected high multiplicity of the solar KK modes, we expect a clear signal above background for $\delta=2$. The important point in testing higher-dimensional effective theory lies in the fact that the relevant combination of the 4-dimensional coupling $g_{4}$ and the total number of KK modes in the kinematically allowed interval $N_{t o t}, g_{4}^{4} N_{t o t}$, turns out to be independent of $M_{*}$ for $\delta=2$ (see below). Assuming no modifications were made on the infrared part of the gauge-boson KK spectrum, this means that non-observation of the KK signal would rule out 2 extra dimensions. Unfortunately, our present experimental setup has no potential to catch a signal above the background for $\delta>2$ modes.

Considering U(1) gauge field propagating in the bulk, the effective 4-dimensional coupling, representing a universal coupling of each KK mode to fermions, can be estimated as [4, 12] as

$$
g_{4}^{2} \gtrsim \frac{M_{*}^{2}}{M_{P l}^{2}}
$$

being thus independent of $\delta$ for a given $M_{*}$. Taking $M_{*} \sim 1 \mathrm{TeV}$, we get $g_{4} \sim 10^{-16}$, and therefore it was argued in [4] that a gauge boson could mediate forces at submillimeter distances $10^{6}-10^{8}$ times stronger than gravity. On the other hand, the zero mode of a new gauge boson (coupled to baryons) can not remain massless since masses larger than $\sim 10^{-4} \mathrm{eV}$ are obligatory in order to comply with fifth force experiments. This means that the gauge symmetry is spontaneously broken in the bulk [4] or on a distant wall [12] by the VEV $v$ of some scalar field. Either case gives $m_{A^{0}}=g_{4} v$. Since the effective theory is valid up to $M_{*}, g_{4} M_{*}$ represents at the same time the upper bound to the mass of the zero mode. By plugging (2), we get $m_{A^{0}} \sim R_{\delta=2}^{-1} \sim 10^{-4} \mathrm{eV}$, and considering the kinematical cutoff (essentially given by thermal energies of electrons in the Sun of order $\sim \mathrm{keV}$ ), we see that only the far-infrared part of the KK spectrum is affected by $m_{A^{0}}$. So we can safely disregard $m_{A^{0}}$ in our considerations. ${ }^{2}$

With the above preliminaries, we are ready to write down the expected number of event in the detector, differential with regard to the gauge boson energy $E$, as

$$
\frac{d N_{b}}{d E}=\frac{d \Phi_{b}}{d E} \sigma_{b+A \rightarrow e+A^{\prime}} N_{\mathrm{Ge}} t
$$

where $N_{\mathrm{Ge}}$ is the number of germanium atoms in the detector, and $t$ is the data collection time.

The differential flux of gauge bosons at Earth in (3), integrated over a standard solar model [16], can be found to be

$$
\begin{aligned}
\frac{d \Phi_{b}}{d E}= & \frac{1}{d_{\odot}^{2}} \int_{\text {Sun }}\left(n_{H}+4 n_{\mathrm{He}}\right) r^{2} d r \int_{0}^{+\infty} d T_{e} \frac{d \sigma_{e+p \rightarrow e^{\prime}+p+b}\left(T_{e}\right)}{d E} \\
& \times \sqrt{\frac{2 T_{e}}{m_{e}}}\left[\frac{2 \sqrt{T_{e}} n_{e} \beta^{3 / 2} \exp \left(-\beta T_{e}\right)}{\sqrt{\pi}}\right],
\end{aligned}
$$

where $d \sigma_{e+p \rightarrow e^{\prime}+p+b}\left(T_{e}\right) / d E$ represents a differential cross section for the bremsstrahlung process in the Sun, and is given by

$$
\frac{d \sigma_{e+p \rightarrow e^{\prime}+p+b}\left(T_{e}\right)}{d E}=\frac{\alpha_{4}}{\pi} \sigma_{\mathrm{Th}} \frac{m_{e}}{T_{e}} \frac{1}{E} \ln \left[\frac{\left(\sqrt{T_{e}}+\sqrt{T_{e}-E}\right)^{2}}{E}\right] N_{\mathrm{tot}}\left(T_{e}\right),
$$

where $\alpha_{4}=g_{4}^{2} /(4 \pi)$. This is just the cross section for $e+p \rightarrow e^{\prime}+p+\gamma$ process, known from QED, with the change $\alpha \rightarrow \alpha_{4}$. As for Eqs.(3-5), a few comments are in order. Firstly, in order to calculate the production rate of gauge bosons in a thermal background analytically, we approximate the Fermi-Dirac electron distribution by a Boltzmann one, i.e., we neglect +1 in front of $e^{E_{e} / T}$, what is perfectly justified because the electron mass is much larger than the

2 Actually, with the present scenario limits on $M_{\star}$ more restrictive than those in 11] can be inferred from strong astrophysical constraints on the emission of new gauge bosons from stars [13, 14] (for a review see [15]). One can easily switch a typical bound on the fine structure constant of forces coupled to electron number, $\alpha_{E} \lesssim 10^{-28}[13$, 14] , to a bound on the fundamental scale by making use of Eq. (2). One obtains, $M_{\star}>10^{4} \mathrm{TeV}$. In contrast, our bound, conditioned by both emission and detection processes, always restricts a quantity $g_{4}^{4} N_{\text {tot }}$ (differently from the emission bound which always restrict $g_{4}^{2} N_{t o t}$ ), which is independent of the fundamental scale for $\delta=2$. Hence our experiment has a potential to rule out the two extra dimensions altogether (see below). 


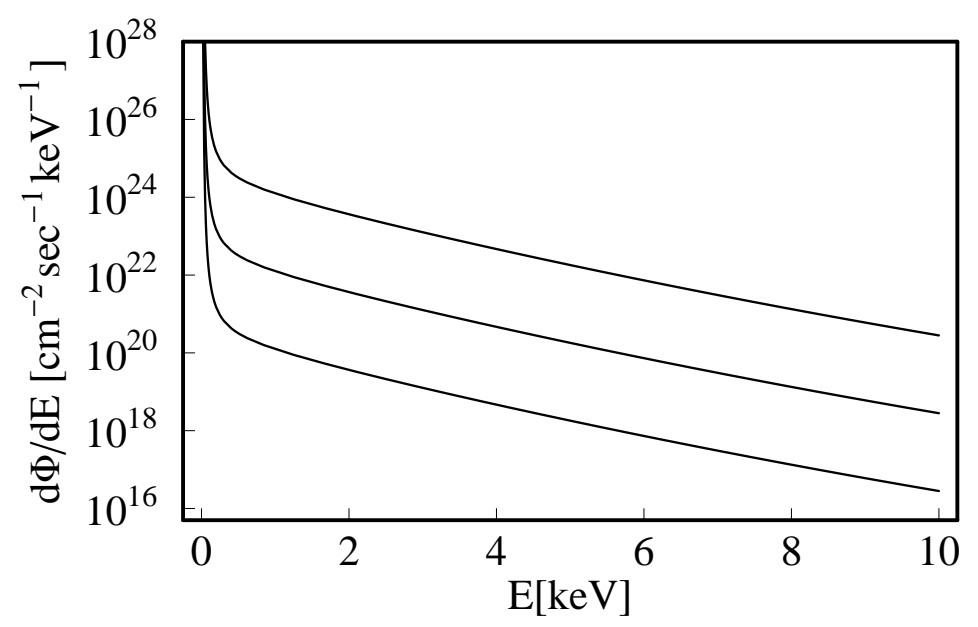

FIG. 1: Energy dependence of the solar flux of KK gauge bosons at Earth, for three values of $M_{*}: 1 \mathrm{TeV}, 10 \mathrm{TeV}$, and 100 $\mathrm{TeV}$ (upper, middle, and lower curve, respectively).

solar temperature. Second, $N_{t o t}\left(T_{e}\right)$ used in (5) captures the effect of massive KK modes, and represents the number of KK modes whose momentum along the extra dimensions is less than the electron kinetic energy $T_{e}$ (see e. g. [17]),

$$
N_{t o t}\left(T_{e}\right)=\frac{S_{\delta-1}}{\delta} \frac{M_{P l}^{2}}{M_{*}^{\delta+2}} T_{e}^{\delta}
$$

where $S_{\delta-1}=(2 \pi)^{\delta / 2} \Gamma(\delta / 2)$ is is the surface of an $\delta$ dimensional sphere with unit radius. We should stress here that we intentionally make use of this approximation (instead of calculating cross section for the production of an individual mode with mass $m$, and then integrating over $m$ 's) in order to show manifestly that by Eqs.(2) and (6)

$$
g_{4}^{4} N_{t o t}\left(T_{e}\right) \sim T_{e}^{\delta} \frac{M_{*}^{2-\delta}}{M_{P l}^{2}}
$$

is independent of $M_{*}$ for $\delta=2$. Further, $\sigma_{\mathrm{Th}}=6.65 \cdot 10^{-25} \mathrm{~cm}^{2}$ denotes the Thomson scattering cross section, $d_{\odot}$ is the distance to the Sun while $n_{e}, n_{H}$ and $n_{H e}$ represent the number densities of electrons, $\mathrm{H}$ and He nuclei in the Sun, respectively. In Fig. 1 we display the flux of KK gauge-bosons at Earth as a function of their energy using some typical values for $M_{*}$.

In a bremsstrahlung process in the Sun most of the gauge bosons will be emitted in the low energy region, as is evident from Fig. 1. For energies of a few keV we estimate that the most sensitive process for detection of gauge bosons will be the boson-electric effect on $\mathrm{L}, \mathrm{M}$ and $\mathrm{N}$ electrons, respectively, of germanium atoms. Therefore the cross section in (3) is calculated from photoabsorption cross section $\sigma_{\gamma+A \rightarrow e+A^{\prime}}$ as

$$
\sigma_{b+A \rightarrow e+A^{\prime}}=\frac{\alpha_{4}}{\alpha} \sigma_{\gamma+A \rightarrow e+A^{\prime}},
$$

with data for $\sigma_{\gamma+A \rightarrow e+A^{\prime}}$ taken from Ref. [18].

After being ejected from the germanium atoms, their energies, together with subsequently emitted X-rays, will be completely absorbed in our large $1.5 \mathrm{~kg}$ HPGe crystal. Detector efficiency relative to 3" $\times 3$ " NaI crystal at $30 \mathrm{~cm}$ was $65 \%$, peak-to-Compton ratio at $1.33 \mathrm{MeV}$ was $60: 1$, and the FWHM at $5.9 \mathrm{keV}$ was $\sim 800 \mathrm{eV}$. Energy calibration was obtained with a set of calibrated radioactive sources. Possible instabilities were controlled on a daily basis, and were less than \pm 1 channel, which is equivalent to $\pm 0.0634 \mathrm{keV}$. Detector was placed inside an iron box with internal dimensions $54 \times 33 \times 33 \mathrm{~cm}^{3}$ and with wall thickness ranging from 16 to $23 \mathrm{~cm}$. The iron was more than 70 years old and was essentially free of ${ }^{60} \mathrm{Co}$ impurities. The box was lined outside with $1 \mathrm{~cm}$ thick lead and background events were reduced by a factor of $\sim 5$. Because most of the gauge bosons are expected to be emitted in the low energy region, special care was taken to check the HPGe detector behaviour for energies below $10 \mathrm{keV}$. The two prominent escape peaks, obtained with radioactive ${ }^{241} \mathrm{Am}$ source, can be seen in Fig. 2. The $3.9 \mathrm{keV}$ peak, originating from the escape of germanium $\mathrm{K}_{\alpha}$ after absorption of niobium $\mathrm{L}_{\alpha}$ X-rays, clearly shows up at $66^{\text {th }}$ channel, and by fitting procedure we find $\mathrm{FWHM} \simeq 663 \mathrm{eV}$. 


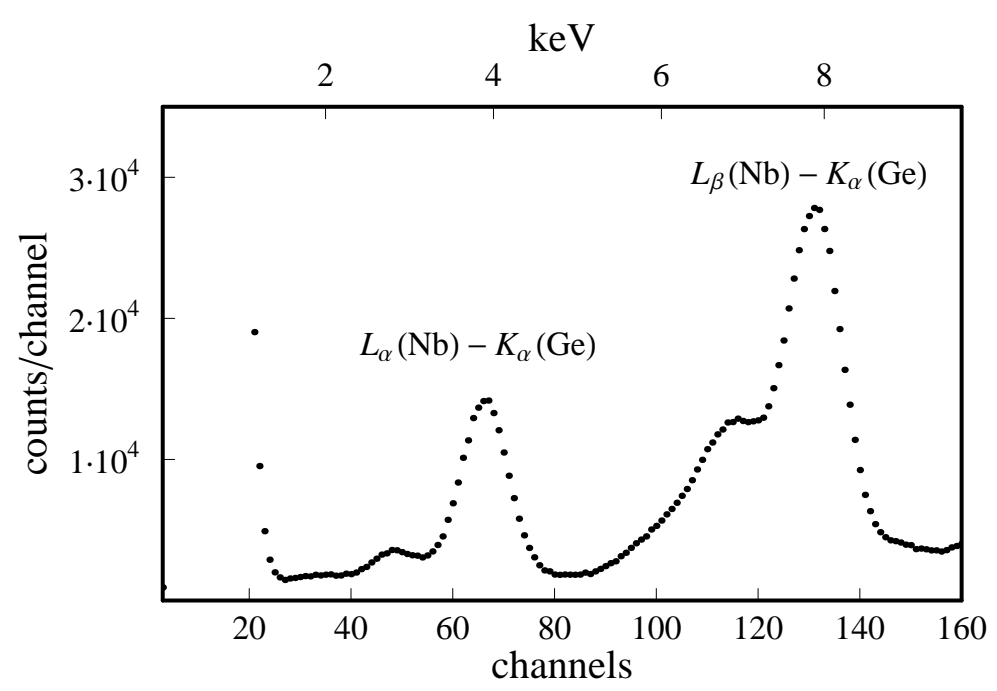

FIG. 2: Low-energy ${ }^{241}$ Am spectra.

The energy spectra below $30 \mathrm{keV}$ accumulated for 202 days are shown with full circles in Fig. 3 , Above a huge electronic noise, which dominates for energies $<1.7 \mathrm{keV}$, there are several visible peaks. Indium $\mathrm{K}_{\alpha}$ and $\mathrm{K}_{\beta}$ peaks show presence of indium which serves as (i) vacuum gasket of ORTEC PopTop detector capsule and (ii) material for making germanium transistors of preamplifier mounted inside the detector capsule. Two escape peaks are due to the escape of germanium $\mathrm{K}_{\alpha}$ and $\mathrm{K}_{\beta} \mathrm{X}$-rays, respectively, after indium $\mathrm{K}_{\alpha} \mathrm{X}$-rays are absorbed in the crystal. The lowest energy peak belongs to copper $8.04 \mathrm{keV} \mathrm{K}_{\alpha} \mathrm{X}$-rays, originating from the copper stick which serves as holder/cooler of the germanium crystal. The most prominent peak, indium $\mathrm{K}_{\alpha}$, served for monitoring of daily spectra stability. All of the above features, during whole measurement, show integrity and stability of total experimental spectrum; there are no systematic errors, i.e. there are only statistical errors.

In Fig. 4 the first 100 channels of Fig. 3 are shown in more details. The full thick line represents the expected KK gauge boson events superimposed on the accumulated energy spectra. The full thin line, which represents fit through the experimental points, is a sum of electronic noise and an almost flat background. Electronic noise is described with a function $y(k)=1.1 \cdot 10^{7} \exp \left[-0.86(k-0.09)^{2} \mathrm{k}^{0.92}\right] k^{6.65}$ and flat part of the spectra with straight line $y_{b}(k)=(33400-31 k) ; k$ is the channel number. Straight line was obtained as the best fit through the experimental data in the 60-100 channels interval, where contribution of KK gauge bosons is expected to be negligible.

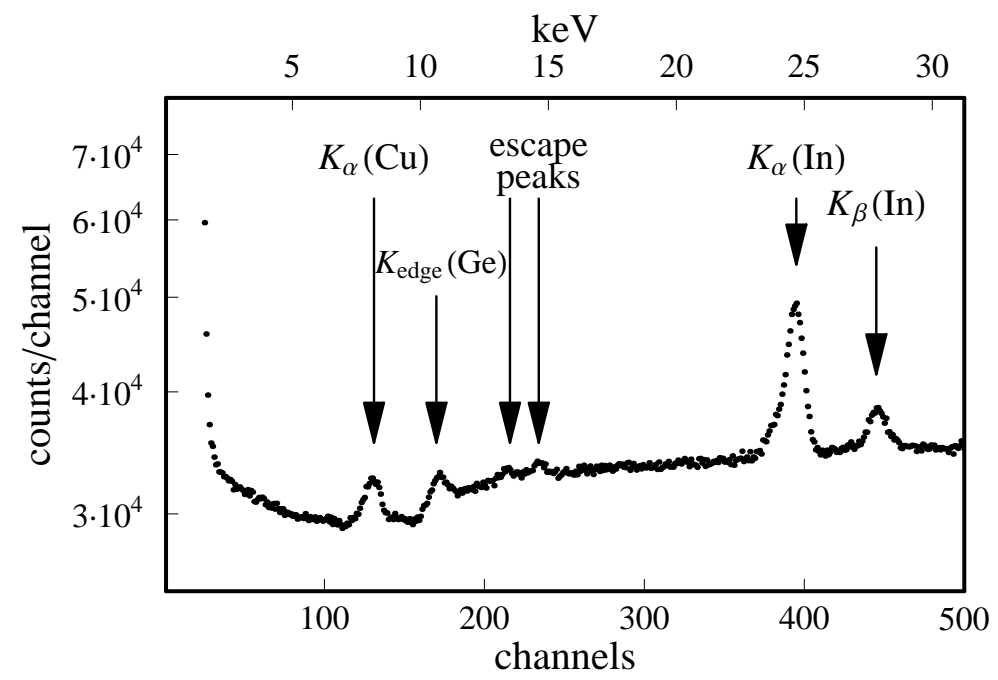

FIG. 3: Energy spectra accumulated in our HPGe detector for 202 days. 


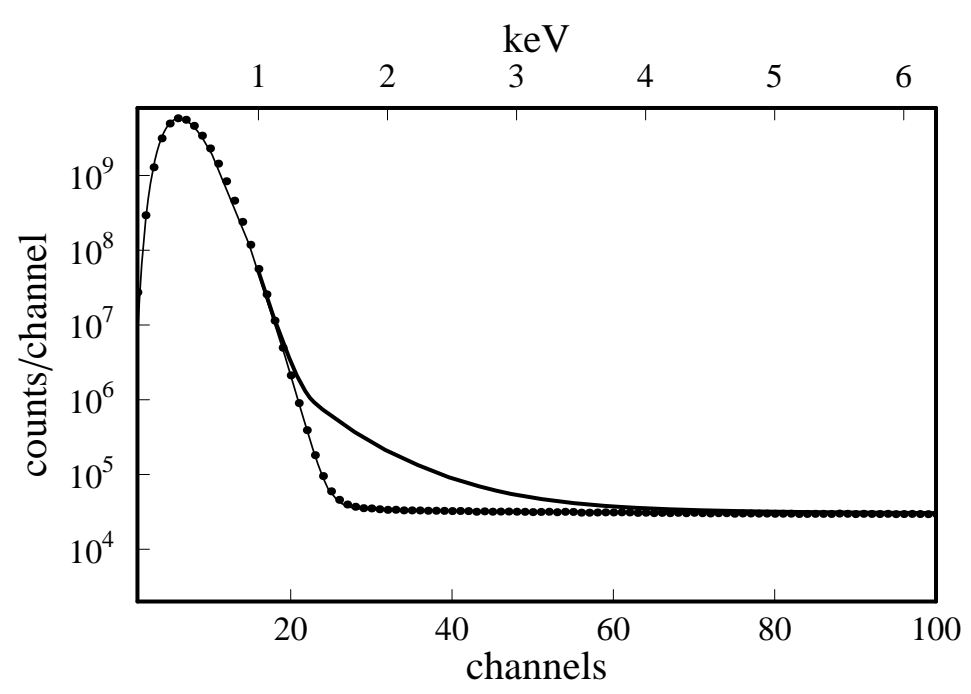

FIG. 4: Number of events in our detector in different energy channels. The expected contribution of KK gauge bosons (thick line), the experimental data (full circles) and fit through the experimental data points (thin line) are shown.

In the energy interval $1.7-3.8 \mathrm{keV}$ the expected number of $\mathrm{KK}$ gauge bosons was $2.7 \cdot 10^{6}$, while for the same energy interval only $1.1 \cdot 10^{6}$ events were detected. At $\sim 1.7 \mathrm{keV}$ the ratio of expected $\mathrm{KK}$ and detected events was approximately 10:1. Given the smallness of $g_{4}$, and the highest mass of the KK excitations in the keV range, in explaining our finding we can safely ignore the possibility of strong attenuation of the gauge bosons in the Sun as well as the possibility that some particles do not survive the journey from the Sun. This means that when this experimental result is interpreted in the context of higher-dimensional low-scale gravity and the ADD proposal, two extra dimensions are definitely ruled out, since by Eq. (7) the effect is expected not to depend on the fundamental scale $M_{*}$. Our result actually supersedes the existing stringent astrophysical bounds for $\delta=2$, which had already been considered to exclude $\delta=2$, if the ADD proposal had something to do with the hierarchy problem.

Finally, we explore a potential of our detector to observe $\delta=3 \mathrm{KK}$ modes. In this case we see from (7) that a dependence on the fundamental scale shows up, but the effect is additionally suppressed by a factor of $T_{e} / M_{*}$ compared with the $\delta=2$ case. In the same time/energy interval as above we have found the expected number of events to be several orders of magnitude below the background. Since this is far below the background, we conclude that the present experimental setup has no potential to explore $\delta>2$ extra dimensions.

In conclusion, we have used a HPGe detector to detect electrons and gammas produced in a U(1)-electric effect when the presumed massive KK modes of the bulk vector field hit the detector from the Sun. Our calculation of the expected number of events assumes that massive KK modes were produced in a bremsstrahlung process in the Sun. With no signal detected above background, and a theoretical prediction that for two extra dimensions the expected number of events in the detector is practically independent of the fundamental scale of gravity, we can definitely rule out the said number of extra dimensions. With the existence of new gauge bosons our result therefore conclusively confirms what a variety of (more model-dependent and thus more uncertain) astrophysical bounds had indicated earlier. In searching for the KK signal from more than two dimensions, we conclude that the limited experimental sensitivity of the present setup has no potential to uncover this possibility.

Acknowledgments. The authors acknowledge the support of the Croatian MSES project No. 098-0982887-2872.

[1] N. Arkani-Hamed, S. Dimopoulos, and G. Dvali, Phys. Lett. B429, 263 (1998).

[2] T. Kaluza, Sitzungdber. Press Akad. Wiss. Berlin (Math. Phys.) 1921, 966 (1921); O. Klein, Z. Phys. 37, 895 (1926).

[3] N. Arkani-Hamed, L. J. Hall, D. R. Smith, and N. Weiner, Phys. Rev. D 62, 105002 (2000).

[4] N. Arkani-Hamed, S. Dimopoulos, and G. Dvali, Phys. Rev. D 59, 086004 (1999).

[5] K. R. Dienes, E. Dudas, and T. Gherghetta, Nucl. Phys. B557, 25 (1999); N. Arkani-Hamed, S. Dimopoulos, G. Dvali, and J. March-Russell, Phys. Rev. D 65, 024032 (2001).

[6] K. R. Dienes, E. Dudas, and T. Gherghetta, Phys. Rev. D 62, 105023 (2000).

[7] I. Antoniadis, N. Arkani-Hamed, S. Dimopoulos, and G. Dvali, Phys. Lett. B436, 257 (1998). 
[8] For a review, see E. G. Adelberger et al., Ann. Rev. Nucl. and Part. Sci. 53, 77 (2003).

[9] G. F. Giudice and A. Sturmia, Nucl. Phys. B663, 377 (2003).

[10] S. B. Giddings and S. Thomas, Phys. Rev. D 65, 056010 (2002); S. Dimopoulos and G. Landsberg, Phys. Rev. Lett. 87, 161602 (2001).

[11] S. Hannestad and G. G. Raffelt, Phys. Rev. Lett. 88, 071301 (2002).

[12] M. L. Graesser, Phys. Rev. D 61, 074019 (2000).

[13] J. A. Grifols and E. Masso, Phys. Lett. B 173, 237 (1986).

[14] J. A. Grifols, E. Masso and S. Peris, Mod. Phys. Lett. A 4, 311 (1989).

[15] G. G. Raffelt, Phys. Rept. 333, 593 (2000).

[16] Solar model BS05(OP) described by J.N. Bahcall, A. M. Serenelli, and S. Basu, Astrophys.J. 621, L85 (2005).

[17] C. Csaki, TASI lectures on extra dimensions and branes, arXiv:hep-ph/0404096.

[18] Berger, M.J., Hubbell, J.H., Seltzer, S.M., Chang, J., Coursey, J.S., Sukumar, R., and Zucker, D.S. (2005), XCOM: Photon Cross Section Database (version 1.3). [Online] Available: http://physics.nist.gov/xcom [2008, February 29]. National Institute of Standards and Technology, Gaithersburg, MD. 